\title{
Review: evidence exists for 33 different treatment options for osteoarthritis of the knee
}

Jordan KM, Arden NK, Doherty M, et al. EULAR Recommendations 2003: an evidence based approach to the management of knee osteoarthritis: report of a Task Force of the Standing Committee for International Clinical Studies Including Therapeutic Trials (ESCISIT). Ann Rheum Dis 2003;62:1145-55.

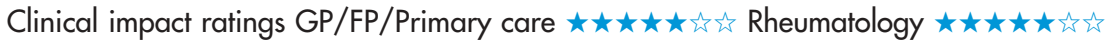

What is the evidence for therapeutic interventions used in knee osteoarthritis (OA)?

\section{METHODS}

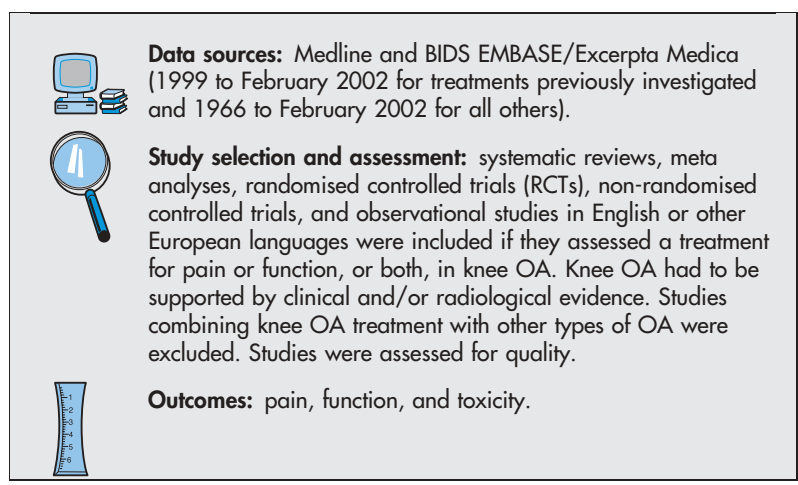

\section{MAIN RESULTS}

103 intervention trials were identified for the overall analysis that involved 33 interventions including non-pharmacological, pharmacological, intra-articular, and surgical treatments for knee OA. Quality scores were highest for studies of glucosamine sulphate and chondroitin sulphate and lowest for surgical studies. 29 of 33 interventions had evidence from $\geqslant 1$ RCT; of the surgical studies, only those of arthroscopy with or without debridement had RCT evidence. RCT evidence exists to support the statement that optimal management of knee OA requires a combination of non-pharmacological and pharmacological treatment options (eg, exercise programmes, physiotherapy, weight loss plus exercise, education, and wedged insoles give added benefit when used with an analgesic or non-steroidal anti-inflammatory drug [NSAID] regimen). RCTs show that (i) education and exercise regimens reduce pain in knee OA and that exercise regimens improve functions, (ii) paracetamol is effective in knee OA treatment and can be taken safely over the long term, (iii) topical NSAIDS and capsaicin are effective and safe for managing knee OA, and (iv) oral NSAIDS are effective for knee OA, although in patients with increased risk of gastrointestinal complications, a cyclooxygenase 2 ( $\mathrm{COX} 2$ ) selective agent or a gastroprotective agent plus oral NSAID should be used (effect size ranges in table). If NSAIDS (including COX 2 selective inhibitors) are contraindicated, ineffective, or poorly tolerated, opioid analgesics with or without paracetamol may be effective. Glucosamine sulphate and chondroitin sulphate have been safe and effective for treating pain and disability in a subset of patients with knee OA. Intra-articular injections of corticosteroid have been effective for flares of knee pain.

\section{CONCLUSION}

Other than surgical studies, most of the 33 identified treatment options for knee OA are supported by evidence from randomised controlled trials.
Effect size ranges $v$ placebo for various knee osteoarthritis interventions*

\begin{tabular}{ll}
\hline Interventions & Effect size ranges $v$ placebo \\
\hline Education & 0.28 to 0.35 \\
Exercise & 0.57 to 1.0 \\
Paracetamol & Not provided in study \\
Topical NSAID & -0.05 to 1.03 \\
Topical capsaicin & 0.41 to 0.56 \\
Oral NSAID & 0.47 to 0.96 \\
\hline *NSAID = non-steroidal anti-inflammatory drug.
\end{tabular}

\section{Commentary}

Tros he review by Jordan et al is commendable for the breadth of management interventions considered. Although the methods used to develop these guidelines are somewhat more rigorous than those used for the American College of Rheumatology, ${ }^{1}$ the subjectivity may still introduce bias. The authors are commended for computing effect sizes. However, the overwhelming majority of trials appear to have no or very few effect size calculations (eg, NSAIDs, COX inhibitors), which raises concerns about the quality of publications or publication bias. Furthermore, the magnitude of some reported effect sizes may be misleading and may lead to unusually strong recommendations for some interventions. This pertains particularly to chondroitin (where the reported effect size ranged from 1.23-1.50), an intervention that was previously subject to the rigorous appraisal of a meta-analysis that found a pooled effect size of $0.78^{2}$ and raised concerns of a possible publication bias. These recommendations suggest that chondroitin is the most effective non-surgical therapy for knee OA. Providing estimates for the range of toxicity from the various experts may have been helpful.

The search strategy focused on pain or function, or both, in knee OA but, surprisingly (given the search strategy), a recommendation was made about structural modification.

Publication bias and other flaws are sufficiently likely that any summary of findings may be misleading. Despite these limitations, a wealth of expertise was used to construct these recommendations, thus they merit widespread attention. Ultimately, the clinician should consider these recommendations in the light of an individual patient's needs because in a heterogeneous disorder like knee OA, evidence-based medicine still requires sound clinical judgement.

David J Hunter, MD, PhD Boston University School of Medicine Boston, Massachusetts, USA

1 American College of Rheumatology Subcommittee on Osteoarthritis Guidelines. Arthritis \& Rheumatism 2001;43:1905-15.

2 McAlindon TE, LaValley MP, Gulin JP, et al. JAMA 2000;283:1469-75. 